\title{
Carbon C 14 Pevonedistat
}

National Cancer Institute

\section{Source}

National Cancer Institute. Carbon C 14 Pevonedistat. NCI Thesaurus. Code C148161.

An orally bioavailable radioconjug ate composed of pevonedistat, a small molecule inhibitor of NEDD8-activating enzyme (NAE), radiolabeled with the radioisotope carbon C 14 , with potential use for evaluating the pharmacokinetic profile of pevonedistat. Pevonedistat binds to and inhibits NAE, which results in the inhibition of tumor cell proliferation and survival. Labeling of pevonedistat with the radioactive tracer carbon C 14 allows for the evaluation of pevonedistat's pharmacokinetic profile, including its absorption, distribution, metabolism, and excretion (ADME). 